\title{
The Weed Plant Detection
}

\author{
V. Geetha, C.K.Gomathy, Y.Padmini Reddy, V.Haripriya
}

\begin{abstract}
The Knowledge about the distribution of weeds within the sector could also be prerequisite for the site-specific treatment. Optical sensors changes to detect vary weed densities and species which can have mapped using GPS data. Weeds are extracted from the pictures that are using the image processing and therefore the report by the form features. The classification supported the features reveal the type and therefore the number of weeds per the image. For the classification the sole maximum of sixteen features out of the eighty-one computed ones is employed. Which enables the optimal distinction of weed classes is used the choice is usually done using processing algorithms, which the speed discriminate of the features of prototypes. If no prototypes are available, clustering algorithms are often used to automatically generate clusters. Within the next step weed classes are often assigned to the clusters. Such procedure aids to select prototypes, which are completed manually. Classes are often identified, that are distinct within the feature space or which are overlapping, and thus not well separable. The clustering is usually utilized in some, less complex cases to work out automatic procedure for the classification. By using the system weed plants are generated. These are differentiating to the results of manual weeds sampling.
\end{abstract}

Keywords: GPS data, automatically generate clusters.

\section{INTRODUCTION}

$\mathrm{T}_{\mathrm{h}}$ the precise application of herbicides in highly infested areas of the sector. Since the spread of weeds is heterogeneous in most of the cases and stable across years (Gerardo et al. 1997, Martens et al. 1998, Gerardo \& CHRISTENSEN 2003), the site-specific weed control can reduce the number of herbicides used. The spraying possesses to be controlled by the particular weed infestation. this manner the selection and therefore the dosage of the herbicides are often optimized for each area of the planet . The first step therefore is to urge information about the distribution of the numerous species. The manual weed sampling is that the time- and cost-intensive and

Manuscript received on April 12, 2021.

Revised Manuscript received on April 26, 2021.

Manuscript published on April 30, 2021.

* Correspondence Author

Dr. V. Geetha, Assistant Professor in CSE Department, SCSVMV Deemed to be University, Kanchipuram, Tamil Nadu.

Dr. CK Gomathy*, Assistant Professor in CSE Department, SCSVMV Deemed to be University, Kanchipuram, Tamil Nadu

Ms. Y.Padmini Reddy, UG CSE Department, SCSVMV Deemed to be University, Kanchipuram, Tamil Nadu.

Ms.V.Haripriya , UG CSE Department, SCSVMV Deemed to be University, Kanchipuram, Tamil Nadu.

(C) The Authors. Published by Blue Eyes Intelligence Engineering and Sciences Publication (BEIESP). This is an open access article under the CC BY-NC-ND license (http://creativecommons.org/licenses/by-nc-nd/4.0/) thus cannot be economic during the broader practice. SLAUGHTER et al. (2008) gives the summary of the techniques for the weed detection and finds, that the robust weed detection remains the primary obstacle toward the commercial development and industry acceptance of the robotic weed control technology. So that, the system was developed to measure the weed infestation.The spatial heterogeneity of weeds has stimulated several weed scientists to review the species distribution of the plants (Wiles et al., 1992; Hazel et al., 1996; the crew \& Cousins, 2001; Gonzalez \& Saved, 2003) and technologies for the weed detection, the spatial weed management and spatial variable applications of herbicides (Gerardo et al., 1997; Christensen \& Hazel, 1998; Piece et al., 1998; Gerard \& the rebel, 2006) \& Hazel, 1998; Piece et al., 1998; Gerard \& the rebel, 2006).

\section{EXISTING SYSTEM}

In existing the tactic, they have use the CNN algorithm for the plant classification. The deep learning method is automatically extracts attributing from two-dimensional plant pictures is proposed generally, the feature maps of the previous layers are converted into informative maps within the conformation layer, and activation functions are provided to form the first feature maps

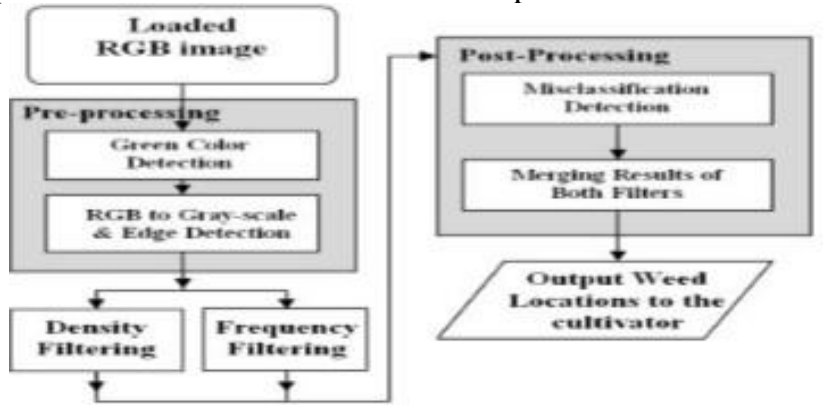

Fig 1: Existing Architecture

\section{PROPOSED SYSTEM}

The machine learning Algorithm prepares the image for the further advanced processing and consists of Loading the image from the source, the colour segmentation, and the edge detection. The operations just like the color segmentation, the edge detection makes the image ready for subsequent operation called the filtering. The filter here is employed for recognizing regions during which edges appear with the frequency during the precise range (the weed frequency range). Here the image after the sting detection within the above step because the input. to use filtering the image has got to be divided into blocks of the certain size.

Published By:

Blue Eyes Intelligence Engineering DOI:10.35940/ijeat.D2454.0410421

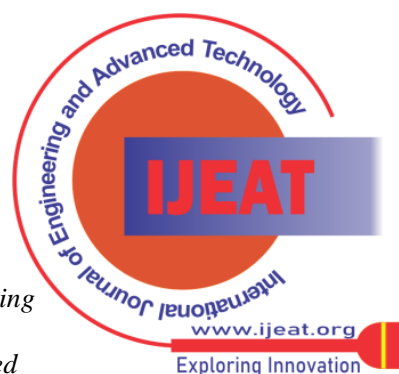
Exploring Innovation 


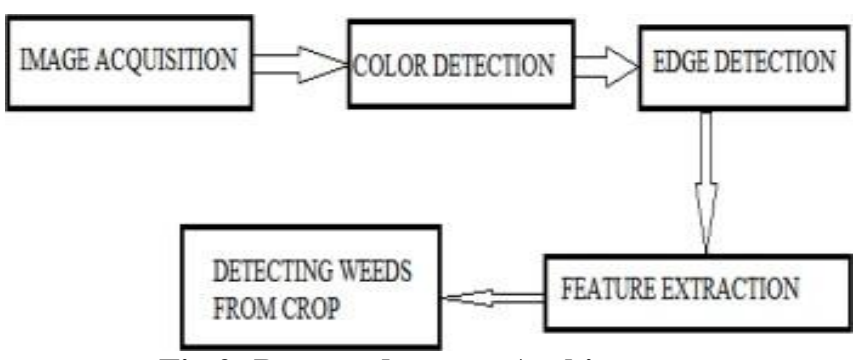

Fig 2: Proposed system Architecture

\section{IMAGE ACQUISITION:}

As mentioned previous, the first step here is acquiring an picture. Here, we examine a field image during a cotton field with the top view throughout the broad day light.

\section{COLOR DETECTION:}

The weed leaves and therefore the crop leaves are green in color. So, color detection cannot be used here. That is why, we opted for edge detection. But, before getting to edge detection we'd like to separate the green colored part from the image. For this, we'd like to regulate the HSV values which are specific for every color HSV table. After this the green colored part is acquired leaving behind the unnecessary soil part. Thus, the acquired green colored part of the image is used for further analysis.

\section{EDGE DETECTION:}

As mentioned earlier, since the colour detection can't be utilized, we went for edge detection. We have many edge detection filters like, Canny, SobelX, SobelY, Laplacian, etc. After verifying the sting detection filters we opted for Laplacian filter which gave the subsequent result, but, before passing a picture through Laplacian filter the noise in a picture must be removed. This is done using Gaussian blur. It not purely removes the noise, it's smoothen the image. The Gaussian blur may be a sort of image blurring filter which may be utilized in one or quite one dimensions.

\section{FEATURE EXTRACTION:}

The final and therefore the crucial step here in weed detection is that the feature extraction. The noticeable feature extraction algorithm in use are SIFT (Scale Invariant Feature Transform) and SURF (Speeded Up Robust Features).

\section{DETECTING WEEDS FROM CROP:}

We now take the help of key points for feature extraction. Key points acts as accurate points of interest which helps in determining the feature of a desired object. This key point identification may be a key step because, in real time the image may get rotated, shrink, translated, or subject to distortion.

\section{IV.FLOW CHART AND WORKING PROCEDURE}

\section{Image Processing}

Image processing maybe the method to changes the picture into the digital form and perform some operations there on, to urge enhanced the image or to extract some useful information from it. It is kind of the signal dispensation through which the input is taken as the picture, like the video frame or the photograph and the output could also be the picture or characteristics related to that picture. Usually Image Processing system involves treating pictures as two-dimensional signals while applying the set signal processing methods to them

\section{Colour Channel Separation}

The RGB picture is separated into individual red, green and blue pictures. This separation is completed to require only the green component of the leaf. The picture is converted only to red, green and blue pictures.

\section{Thresholding}

Thresholding may be a simple and effective technique wont to partition the image into background and foreground. Here "graythresh" is used to change the green image into global image threshold by using Otsu's method.

\section{Otsu Thresholding}

OTSU's method performs the clustering-based the picture threshold or the reduction of the gray level picture to the binary picture. The algorithm shows that the picture contains two classes of following pixels the bi-modal histogram, it then calculates the optimum threshold separating the 2 classes, so that, their combined the spread (the intra-class variance) is less, or equivalently (because the sum of pairwise squared distances is the same), in the order that their inter-class variance is more. The extension of the first method to the multi-level threshold is mentioned because the multi OSU method. Morphology may be a broad set of picture processing operations that process pictured in supported shapes. In morphological operation, erosion and dilation is done. Dilation means growing image regions. Dilation is ended with the help of imdilate function. Erosion means shrink image regions. Erosion is done using imerode function.

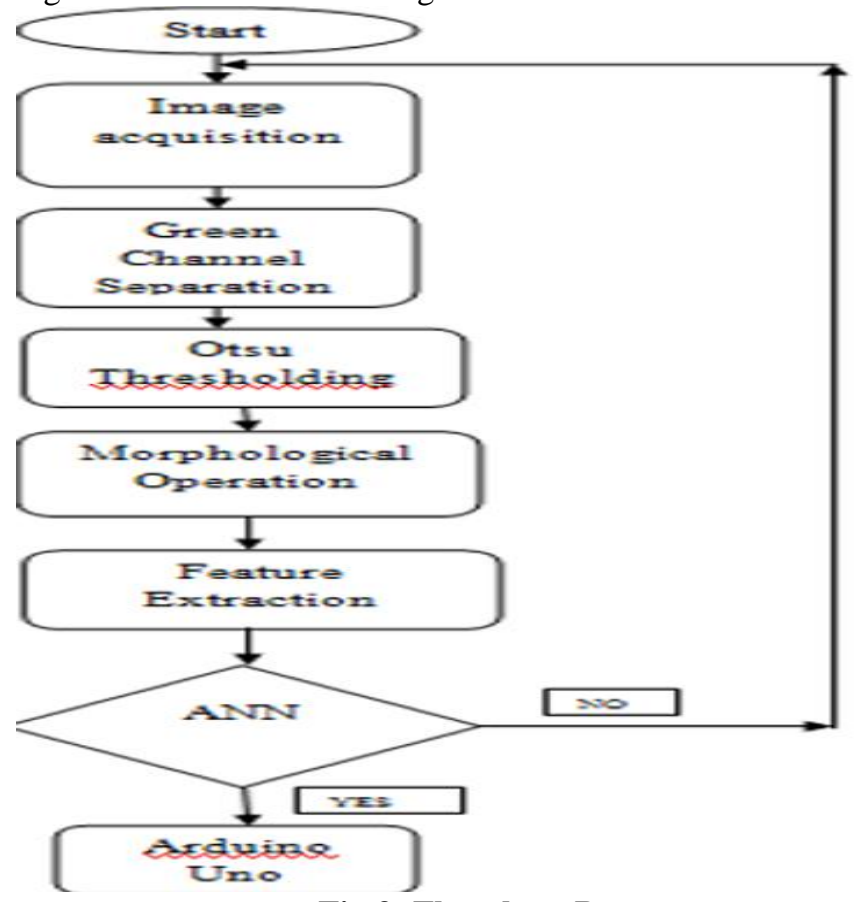

Fig 3: Flowchart Process

The software tool we used here is Python3.6.7.

Python is the prominent languages used for Image processing. It includes csome packages that make Image processing easy to implement.

Published By:

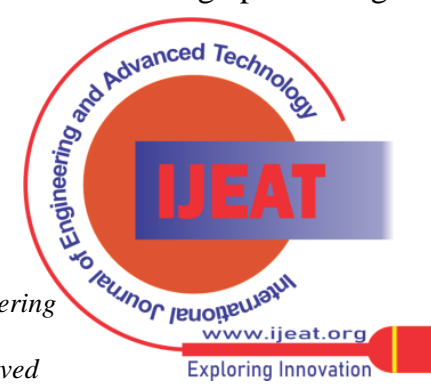


We took help of OpenCV which is an open platform for certain programming languages like $\mathrm{C}, \mathrm{C}++$, JAVA, and Python. We have installed certain packages such as Numpy, PyWavelet, Matplotlib, pydot etc. All this work was done by us on a Windows operating system with an inbuilt Microsoft Visual Studio.

\section{V.TEST RESULTS}

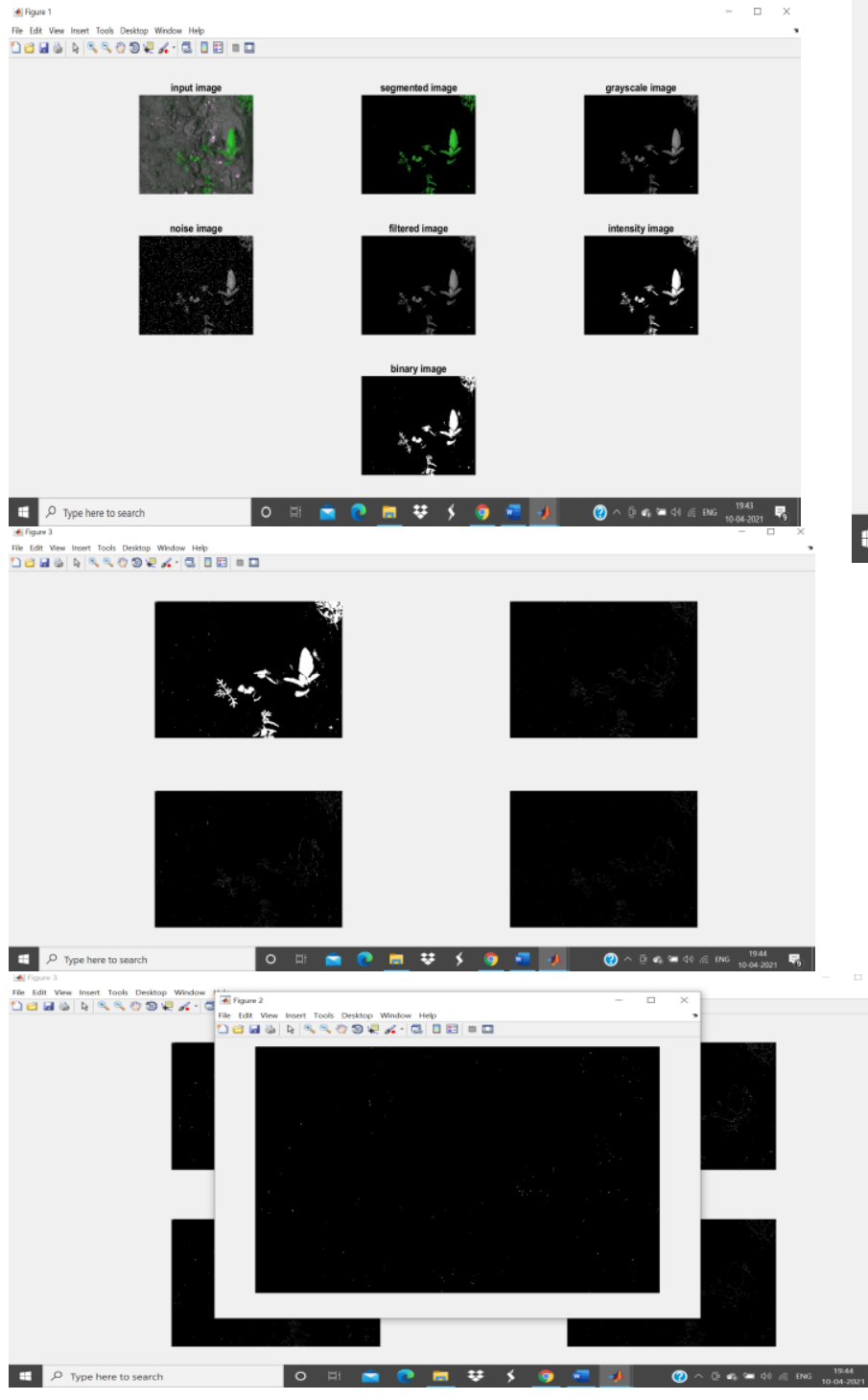

4
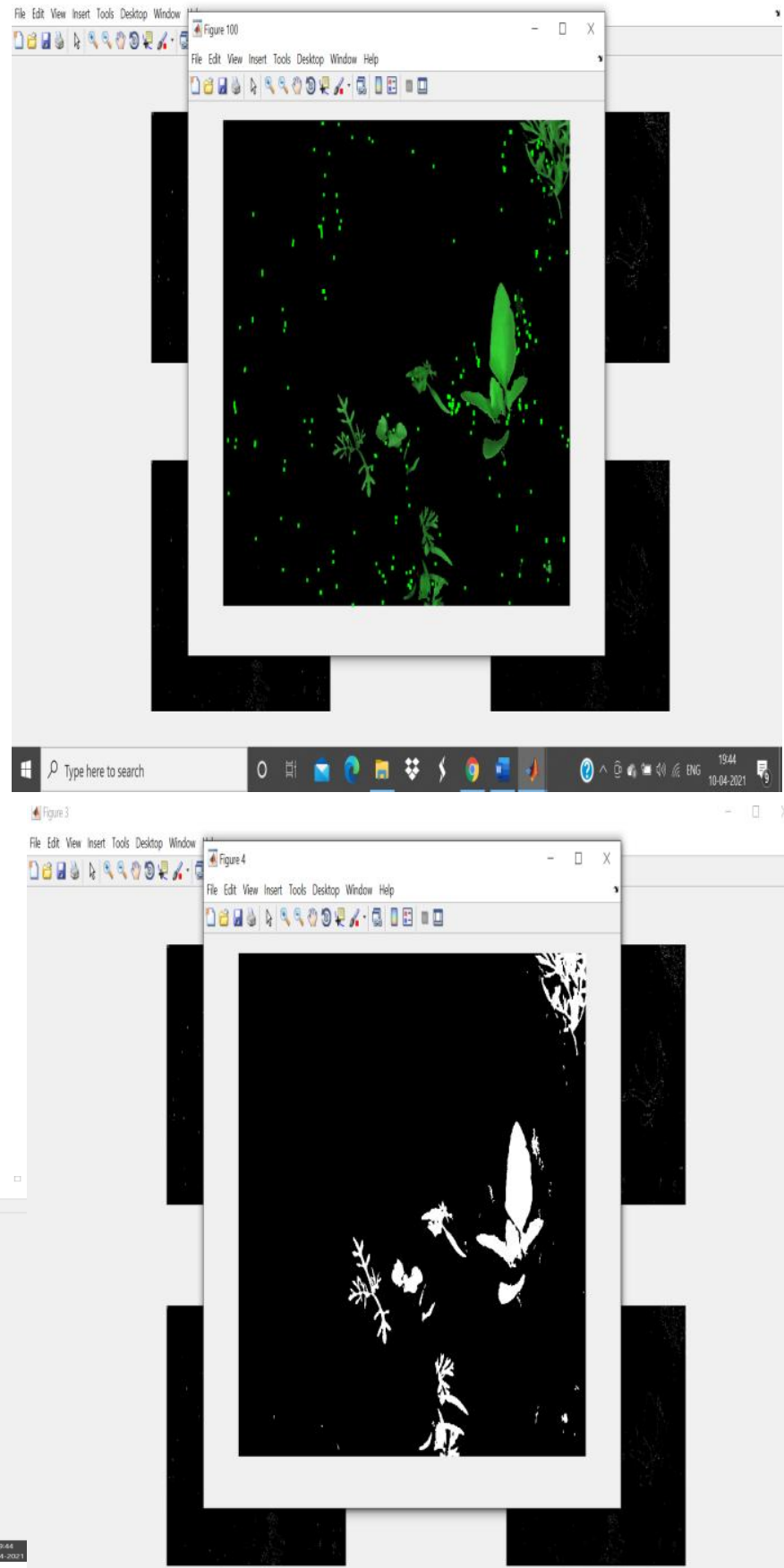

4

Tipe here toserch

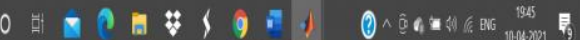

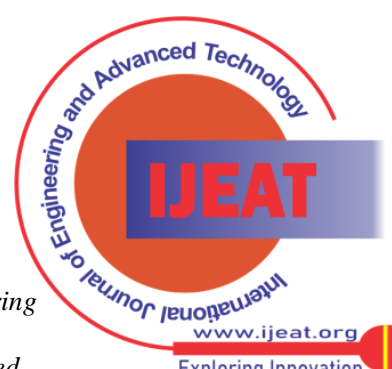




\section{The Weed Plant Detection}

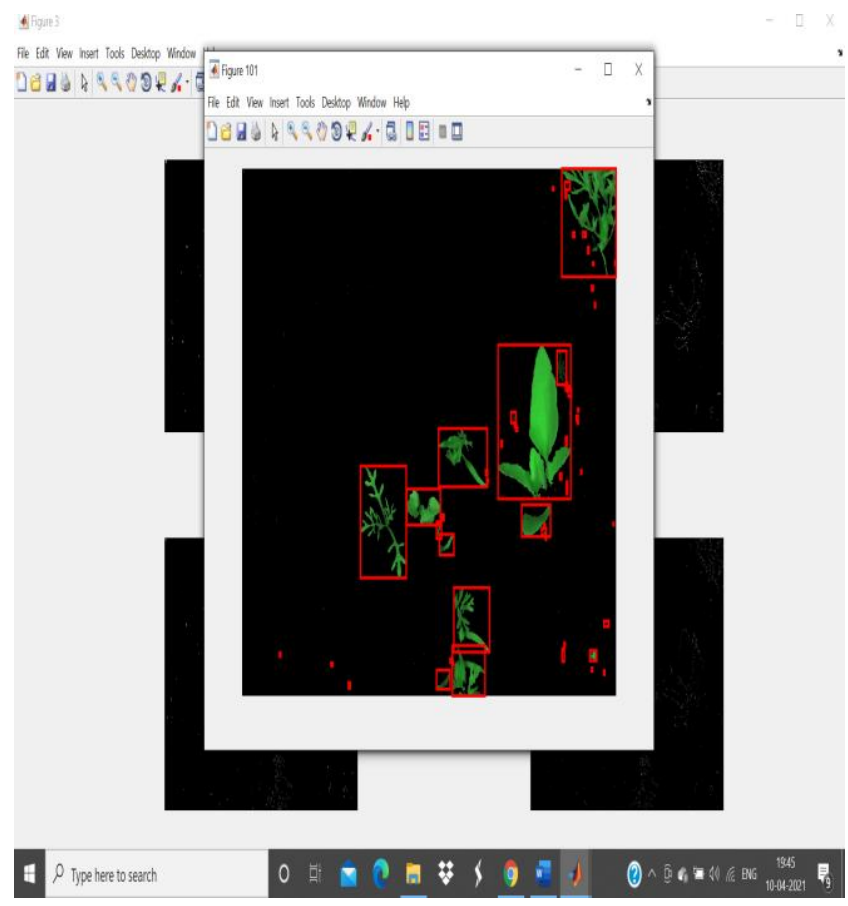

\section{CONCLUSION}

In this system, we've developed how by which we'll detect weed using Image processing. Thanks to the use of our system, we'll detect and filter weed affected area from the crop plants. the rationale for developing such system is to identify and reuse weed affected area for more seeding. These specific areas are often considered for further weed control operations, resulting in more production.Employing the processes like segmentation, feature extraction and clustering are often used to interrogate images of the crops. there's a requirement to select the foremost appropriate techniques to assist decision-making. The image processing techniques are used across an enormous range of agricultural production contexts. The accuracy of classification varies relying on the algorithms resolution of images and limitations of image acquisition.

\section{REFERENCE}

1. Martin Abadi, Ashish Agarwal, Paul Barham, Zhifeng Chen, Craig Citro, Greg S. Corrado, Andy Davis, Jeffrey Dean, Sanjay Ghemawat, Michael Isard, Yangqing Jia,Rafal Jozefowicz,Dandelion Mane ,Rajat Monga, Sherry Moore, Derek Murray, Mike Schuster, Jonathon Shlens, Benoit Steiner, Ilya Sutskever, Fernanda Viegas, Pete Warden, Martin Wicke, and Yuan Yu. TensorFlow: Largescale machine learning on heterogeneous systems, 2014. Software available from tensorflow.org.

2. L'eon Bottou. Large-scale machine learning with stochastic gradient descent. In Proceedings of COMPSTAT'2010, pages 177-186. Springer, 2010.

3. Zheng Cao, Jose C Principe, Bing Ouyang, Fraser Dalgleish, and Anni Vuorenkoski. Marine animal classifying using combined $\mathrm{cnn}$ and handdesigned image features. In OCEANS'15 MTS/IEEE Washington, pages 1-6. IEEE, 2015.

4. Qiang Chen, Rahil Garnavi, and Xi Liang. Ibm research australia at lifeclef2014: Plant identification task. In CLEF (Working Notes), pages 693-704, 2014. [5] Fran,cois Chollet et al. Keras. https://github.com/fchollet/keras, 2015.

5. EU Directive. 128/ec of the ecu parliament and of the council of 21 october 2009 established a framework for community actions to realize the sustainable use of pesticides. EU, Brussels, 2009.

6. Herv'e Go"eau, Alexis Joly, Pierre Bonnet, Souheil Selmi, Jean-Fran, cois Molino, Daniel Barth'el'emy, and Nozha Boujemaa. LifeCLEF Plant Identification Task 2014. In L. Cappellato,

7. Dr.C K Gomathy, Article: A Semantic Quality of Web Service Information Retrieval Techniques Using Bin Rank, International Journal of Scientific Research in Computer Science Engineering and Information Technology ( IJSRCSEIT ) Volume 3 | Issue 1 | ISSN : 2456-3307, P.No:1563-1578, February-2018

8. Dr.C K Gomathy, Article: A Web Based Platform Comparison by an Exploratory Experiment Searching For Emergent Platform Properties, IAETSD Journal For Advanced Research In Applied Sciences, Volume 5, Issue 3, P.No-213-220, ISSN NO: 2394-8442,Mar/2018

9. Dr.C K Gomathy, Article: A Study on the Effect of Digital Literacy and information Management, IAETSD Journal For Advanced Research In Applied Sciences, Volume 7 Issue 3, P.No-51-57, ISSN NO: 2279-543X,Mar/2018

10. Dr.C.K.Gomathy, A.V.Sripadh Kaustthub, K.Banuprakash, Article: An Effect of Big Data Analytics on Enhancing Automated Aviation , International Journal Of Contemporary Research In Computer Science And Technology (Ijcrcst) E-Issn: 2395-5325 Volume 4, Issue 3,P.No-1-7.March -2018

11. Dr.C K Gomathy, Article: A Semantic Quality of Web Service Information Retrieval Techniques Using Bin Rank A Cloud Monitoring Framework Perform in Web Services, International Journal of Scientific Research in Computer Science Engineering and Information Technology IJSRCSEIT | Volume 3 | Issue 5 | ISSN : 2456-3307,May-2018

12. Dr.C K Gomathy, Article: Supply chain-Impact of importance and Technology in Software Release Management, International Journal of Scientific Research in Computer Science Engineering and Information Technology ( IJSRCSEIT ) Volume 3 | Issue 6 | ISSN : 2456-3307, P.No:1-4, July-2018

13. Dr.C K Gomathy, Article: A Scheme of ADHOC Communication using Mobile Device Networks, International Journal of Emerging technologies and Innovative Research ( JETIR ) Volume 5 | Issue 11 | ISSN : 2349-5162, P.No:320-326, Nov-2018

14. Dr.C K Gomathy, Article: A Study on the recent Advancements in Online Surveying, International Journal of Emerging technologies and Innovative Research ( JETIR ) Volume 5 | Issue 11 | ISSN : 2349-5162, P.No:327-331, Nov-2018.

15. Dr.C.K.Gomathy,P.Sarvani Divya jyothsna,M.Srimayi, Article: A study on the Mobile Application Advancements in Anti-Ragging, SSRG International Journal of Computer Science And Engineering(SSRG-IJCSE)-Volume 6 issue 3,March 2019.

16. C.K.Gomathy and Dr.S.Rajalakshmi.(2014),"A Software Quality Metric Performance of Professional Management in Service Oriented Architecture", Proceedings of ICCTET'14, organized by Akshaya College of Engineering, Coimbatore. Archived in IEEE Xplore Digital Library, July 2014,ISBN:978-1-4799-7986-8

17. C.K. Gomathy, V. Geetha, Venkata Nagendra, Venkata Sai Ram, Article: The Secured Proficient Smart Electronic Voting System, International Journal of Engineering and Advanced Technology (IJEAT) ISSN: 2249 - 8958, Volume-9 Issue-4, April 2020

18. V.Geetha, C.K.Gomathy, P.Manojkumar, N.S.L.S.V.Manohar, Article: The Sentimental Analysis for E-Commerce Application, International Journal of Engineering and Advanced Technology (IJEAT) ISSN: 2249 - 8958, Volume-9 Issue-5, June 2020

19. C K Gomathy,V Geetha,P.S.lavakumar,K.V.Rahul,The Efficient Automatic Water Control Level Management Using Ultrasonic Sensor, International Journal of Computer Applications (0975 - 8887) Volume 176 - No. 39, July 2020

20. V. Geetha, C.K. Gomathy, Maddu Pavan Manikanta Kiran, Gandikota Rajesh, The Implementation of Fleet Monitoring System using Mobile based Software, International Journal of Engineering and Advanced Technology (IJEAT) ISSN: 2249 - 8958, Volume-9 Issue-4, April 2020.

21. C K Gomathy, Student Location Tracking Inside College Infrastructure, International Research Journal of Engineering and Technology (IRJET) e-ISSN: 2395-0056 Volume: 07 Issue: 06 | June 2020

Published By:

Blue Eyes Intelligence Engineering

\& Sciences Publication

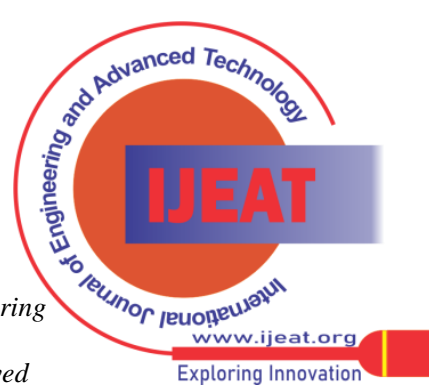




\section{AUTHORS PROFILE}

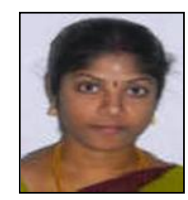

Dr.V.Geetha, is assistant professor in computer science and engineering at Sri Chandrasekharendra SaraswathiViswa Mahavidyalaya ,Enathur ,Kanchipuram, India. Her area of intrest lies in Java Programming,Software Quality Assurance,Computer System Architecture Domain.

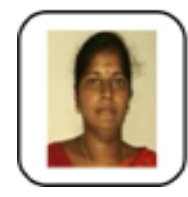

Dr. C.K.Gomathy is assistant professor in computer science and engineering at Sri Chandrasekharendra Saraswathi Viswa Mahavidyalaya, Enathur, Kanchipuram, India. Her area of intrest lies in Software Engineering,Web Services, KnowledgeManagement Domain.

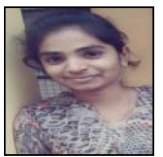

Y.Padmini Reddy, student, B.E. Computer Science and Engineering, SCSVMV University .

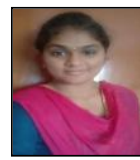

V.Haripriya, student, B.E. Computer Science and Engineering SCSVMV University.

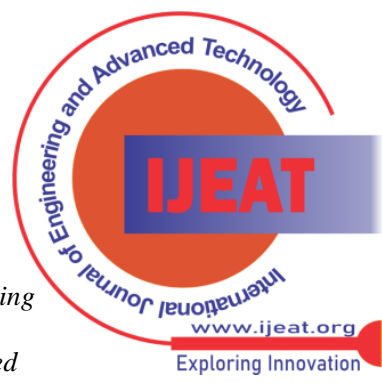

